\title{
Review of HRP Positions
}

\section{OR I S E}

Center for Human Reliability Studies Oak Ridge Institute for Science and Education Operated by Oak Ridge Associated Universities For the U.S. Department of Energy 
Oak Ridge Associated Universities (ORAU) is a university consortium leveraging the scientific strength of major research institutions to advance science and education by partnering with national laboratories, government agencies, and private industry. ORAU manages the Oak Ridge Institute for Science and Education for the U.S. Department of Energy.

The Oak Ridge Institute for Science and Education (ORISE) is a U.S. Department of Energy institute focusing on scientific initiatives to research health risks from occupational hazards, assess environmental cleanup, respond to radiation medical emergencies, support national security and emergency preparedness, and educate the next generation of scientists. ORISE is managed by Oak Ridge Associated Universities.

The Center for Human Reliability Studies (CHRS) provides a vehicle for achieving quality-oriented program support for DOE through research and analysis, technical guidance, and operational support in all areas of human reliability relating to occupational medicine, facility safety, personnel security, and the DOE Human Reliability Program. CHRS also conducts research on issues involving personnel reliability.

This document was produced under contract number DE-AC05-06OR23100 between the U.S. Department of Energy and Oak Ridge Associated Universities. 


\title{
Review of HRP Positions
}

\author{
Prepared for \\ Office of Security Policy \\ Office of Health, Safety and Security \\ U. S. Department of Energy \\ Washington, D.C.
}

\begin{abstract}
by
Center for Human Reliability Studies

Oak Ridge Institute for Science and Education

Oak Ridge, Tennessee
\end{abstract}

ORISE 07-0023

January 2007 



\section{Introduction}

The Department of Energy (DOE) Human Reliability Program (HRP), published as 10 CFR Part 712 , is currently being reviewed and revised to address concerns identified during its implementation. Although these "page changes" primarily incorporate clarification of terms and language, the following discussion relates to broadening the definition of positions that require HRP certification that is found in $\$ 712.10$.

Because the program has matured, an evaluation of additional positions to be included in the HRP is germane. Since the current HRP is used throughout the DOE complex to mitigate the insider risk from active to passive, other positions that have the potential to significantly impact our national security must be considered for inclusion into the program.

Both physical security and the motivation and commitment of employees are required to protect DOE's critical information and material assets. As promulgated, the HRP focuses on individuals in positions that afford access to Category I special nuclear material (SNM) and relevant safeguards and transportation information. Those positions are well covered by the program as it currently stands. However, many other positions occupied by individuals who create or control the flow of sensitive information or protect other assets are not included in the HRP. Examples include executive protection services agents, who contribute to the safety and security of the Secretary of Energy, foreign dignitaries, and heads of state; systems administrators having access to classified information systems; and individuals who have access to chemical and biological agents or the detection systems for those agents.

Since safety and security are heavily dependent on the willingness of each individual to comply with rules, regulations, and procedures, the HRP certification and recertification requirements seek to identify conditions or circumstances that could result in noncompliance. A careful analysis of the physical and mental characteristics of the individual, past behavior, and information obtained during the certification or recertification process are all critical elements of detecting these factors. The areas considered to be significant indicators of potential risk are:

Alcohol and substance abuse: Problems with self control and the ability to conform to legal, social, and domestic norms may be indicated by alcohol and/or substance abuse. 
Financial responsibility: How individuals manage money can reveal much about their self control and vulnerability to coercion and blackmail.

Honesty: Individuals who are honest in their daily lives are more likely to comply with organizational demands and perform in a safe, reliable, and trustworthy manner.

Arrests and convictions: Failure to comply with societal demands, which leads to problems with the law, could reflect broader problems with conformance.

Social conformance: The ability and willingness to conform to rules, regulations, and procedures is crucial to safety and security in DOE facilities. Like arrests and convictions, other forms of social nonconformance greatly increase the risk that individuals may compromise the safety and security of the organization.

Vulnerability: Susceptibility to coercion can result from a variety of financial or personal conditions.

Psychological stability: Safety and security compliance demands that an individual be in control of thoughts, motives, and impulses. Mental stability may affect control and ultimately the ability of the individual to conform.

An individual who works in a position requiring HRP certification is evaluated on an ongoing basis, increasing the probability that conditions and circumstances indicating a concern will be detected. HRP-certified individuals may hesitate to jeopardize their positions by revealing such things as arrests, illnesses, or changes in domestic status or mental condition. Yet, in a one-on-one interview with a psychologist or other medical professional and a DOE personnel security review may reveal information that could indicate a change in risk status. It should also be noted that HRP certification, which serves as a constant reminder of unacceptable behaviors and conditions that could result in a loss of access or certification, could thereby have a deterrent effect.

\section{Continuous observation}

The HRP is a preemptive program, designed to detect concerns before safety and security are compromised. The HRP process of continuous observation is designed to identify individuals who represent a potential reliability risk and ensure that DOE's most sensitive materials and information are protected. 
The HRP formal evaluation process offers a comprehensive means of identifying behaviors and conditions that may indicate safety and security risks. The basic elements of continuous evaluation are performed for certification and then at least once every 12 months thereafter for recertification, but observation is conducted on an ongoing basis. Elements of observation include:

Supervisory review: An HRP-certified individual's supervisor has daily contact with the individual and is in a position to observe changes in behavior. HRP supervisors are trained in observation skills, and are in a position to make reports and referrals before unusual behavior becomes a safety or security reliability issue. Each year for HRP recertification, the supervisor formally evaluates each HRP-certified individual and makes a recommendation regarding certification.

Medical assessment: Facility occupational health professionals perform a comprehensive evaluation of each HRP-certified individual at 12-month intervals. An assessment may also be performed for self-referrals and management concerns. The occupational health office also tracks medication use and evaluates both prescription and over-the-counter medications against the job task analysis to determine the reliability risks of each individual's medication use. HRP-certified individuals are required to report to the occupational health office for a return-to-work evaluation after a five-day period of absence. Medical monitoring of rehabilitative programs is also provided.

Random drug and alcohol testing: HRP candidates must successfully complete drug and alcohol tests before they receive certification. HRP-certified individuals are then subject to drug and alcohol tests on a random basis. Random tests must be performed at least once in every 12 -month period. These tests serve both passively, as a deterrent, and actively, as a means to identify drug and alcohol use among HRP-certified individuals.

Management review: Every 12 months for HRP recertification, the manager performs a careful evaluation of the results of the supervisor review, medical assessment, and random drug and alcohol testing, and provides a recommendation that the individual either be recertified or removed from the HRP. This review provides objective oversight of the continuous observation process.

DOE personnel security review: DOE personnel security specialists perform a review of each HRP candidate and HRP-certified individual's personnel security file and evaluate the 
information found on Part 2 of the Questionnaire for National Security Positions. Because this information can indicate potential or ongoing problems with reliability, an annual review is invaluable in detecting security concerns.

Instruction: Initial instruction is provided to all HRP candidates, followed by annual refresher instruction for recertification. This instruction is comprehensive and covers all elements of the HRP. Educational information is also available on other aspects of the HRP such as the observation and reporting of unusual behavior, reporting medication use, and identifying safety and security concerns.

Individual awareness: HRP-certified individuals have a role in the continuous observation process and are required to report reliability concerns in both themselves and others.

This regular and formal process of continuous observation is vital to the success of the HRP in early identification of safety and security concerns. All elements of continuous observation are integrated into a comprehensive program. Therefore, extending HRP certification to other critical positions enhances national security and the safety of employees and the public by ensuring that individuals in these positions meet the highest standards of reliability and physical and mental suitability.

\section{Current HRP positions}

Section 712.10 of the HRP rule currently defines a position requiring HRP certification as one that:

(1) Affords access to Category I SNM or has responsibility for transportation or protection of Category I quantities of SNM;

(2) Involves nuclear explosive duties or has responsibility for working with, protecting, or transporting nuclear explosives, nuclear devices, or selected components;

(3) Affords access to information concerning vulnerabilities in protective systems when transporting nuclear explosives, nuclear devices, selected components, or Category I quantities of SNM; or

(4) Is not included in paragraphs (a)(1) through (3) of this section but affords the potential to significantly impact national security or cause unacceptable damage and is approved pursuant to paragraph (b) of this section. 
These categories of positions are taken from the former PSAP [10 CFR Part 710 (Subpart B)] and PAP (10 CFR Part 711) rules, which were combined to form the basis for the HRP. The PSAP and PAP were supplanted by the HRP rule when it was published on January 23, 2004 as 10 CFR Part 712.

The HRP allows additional positions to be nominated after an assessment process that includes an evaluation of the risks the position poses for the operational program. If the analysis shows that more restrictive physical, administrative, or other controls could be implemented that would prevent the position from being designated an HRP position, those controls will be implemented, if practicable. Any nominations must be approved by the NNSA Administrator or designee, the Chief Health, Safety and Security Officer or designee, or the appropriate Lead Program Secretarial Officer or designee.

The positions described below do not currently require HRP certification but have the potential to significantly impact the DOE mission and national security. DOE's overall security posture would be strengthened by including these positions with those currently designated in the HRP.

\section{Proposed HRP positions}

Although a provision exists in the HRP to designate positions other than those specified in $\$ 712.10(\mathrm{a})$, in practice this provision has not been widely employed. Certain positions, which should require HRP certification because of the risk to safety and security, are not included in the HRP rule. Failure to incorporate these positions poses a risk to the safety and security of DOE facilities and our national security. It is therefore proposed that certain new categories of positions be added to those currently listed in $\$ 712.10(\mathrm{a})$. A brief justification accompanies each category.

\section{Weapons designers}

Positions within the DOE weapons program, specifically weapons designers with access to weapons data categories Sigma 0 through 15, do not currently require HRP certification. These tasks, however, entail specialized knowledge of weapons design and operations data that requires the highest level of reliability. Such individuals have knowledge not only of current weapons designs but also entire weapons systems. That information could be invaluable to someone wishing to harm the United States or its interests. The security of that knowledge and information is subject to the reliability and trustworthiness of the designers. 
Reviewing a weapons designer's alcohol and drug use, social conformance, financial status, and psychological stability may reveal the risk potential of that individual. Drug and alcohol abuse can interfere with the ability to control impulses and behavior and result in a failure to comply with the rules and regulations regarding the protection of classified information. An even greater risk lies in the stress-money connection. Domestic conditions can often lead to financial problems, also producing a significant amount of stress. Such pressure can increase the likelihood that an individual might sell valuable information, believing that the money will reduce the stress and solve the problem. HRP certification makes it more likely that an occupational health professional will be in a position to explore those issues and determine whether a risk is present. When identified, such information is provided to DOE security elements for evaluation of the risk factors.

HRP certification and recertification evaluations not only provide an opportunity to discover those who may pose a safety or security concern, but also to emphasize the need for security and vigilance in HRP-certified individuals. Those working with classified weapons information and materials can sometimes become complacent about protection procedures. Questions about arrests, alcohol and drug use, family situation, financial condition, as well as psychological testing, not only provide valuable information to HRP certification decision makers but also underscore the vital role each HRP-certified individual plays in protecting safety and security. Being subject to the HRP recertification process thus underscores the importance of protecting DOE assets and highlights many factors that can interfere with that process.

At a minimum, positions requiring Sigmas 14 and 15 should be included in the DOE HRP. Sigma 14 is the category of information concerning the vulnerability of nuclear weapons to deliberate unauthorized nuclear detonation. Sigma 15 is the category of information concerning the design and function of nuclear weapons control systems, features, and their components. This includes use control information for passive and active systems. Both Sigma categories should be designated as HRP positions because they include extremely sensitive information that requires the highest level of reliability. Sigma designations 1-13 should also be included in the HRP, however individual Sigma evaluations could be conducted to determine the necessity of their inclusion in the HRP.

\section{Executive protection services}

Agents in the executive protection services, responsible for the safety and security of the Secretary of Energy, also at times have access to the President of the United States, foreign dignitaries, heads 
of state, and the security plans and procedures necessary to ensure the safety of these important personnel.

Members of the protection services have access to information that could be useful to anyone planning to harm government executives. The HRP continuous evaluation process can produce information about personal issues or medical conditions that may affect an agent's ability to respond to a situation. Additionally, individuals who are under stress, abusing drugs and/or alcohol, or subject to other conditions or circumstances may not be able to perform their duties in a safe and reliable manner.

The HRP would provide an opportunity to assess the current functioning of executive protection services agents. While in the field, success in performing their duties depends on constant vigilance and attention. Stress, mental conditions such as depression or anxiety, and domestic problems can all affect cognitive functioning. The HRP medical assessment may identify conditions including lack of visual acuity; impaired hearing; neuromuscular impairment; cardiovascular, endocrine, cerebravascular, or other neurologic diseases; or the use of medications that could affect judgment or ability to perform assigned duties in a reliable and safe manner. The HRP medical assessment provides important information regarding an individual's physical and mental status, which are critical elements to those in the executive protection services.

Although executive protection services personnel are carefully selected and vetted, their circumstances may change with time. The HRP is designed to continuously evaluate individuals in positions where unreliable behavior or circumstances could pose a serious threat to safety and security. The HRP recertification process can ensure that the qualities which resulted in initial selection are still present, and that the agents remain dedicated to the safety and security of those they protect. Executive protection services personnel should therefore be added to the positions requiring HRP certification to ensure that their physical and mental suitability are at the highest level.

\section{Systems administrators}

Insider compromise of physical security measures can be a risk in DOE facilities. Many physical security measures are controlled by computer programs and hardware. In addition, virtually all sensitive information is contained in digital records. Systems administrators have the potential to 
divert information and conceal such diversion. Personnel in those positions know how the software and hardware works, and thus they understand its vulnerability.

Systems administrators can have access to highly classified materials, information about vulnerabilities, protection force deployment, SNM movements, and storage of classified data that could be valuable to persons wishing to harm the organization or nation. A skilled administrator could bypass the protections and gain access to the information without being detected. Computer security, to a considerable extent, still depends on the dedication of those charged with its development and maintenance.

Managing computer systems requires that individuals comply with the procedures designed to protect information from corruption or diversion. Anything that could affect the willingness to comply increases the risk potential. There are many ways in which the systems could be compromised, including the unauthorized transfer of classified information into an unclassified system. Such an activity could be caused by domestic stress, medical bills, or other financial demands. Coercion because of conflicting loyalties, illegal activities, or the abuse of drugs or alcohol is also a concern.

Disgruntled workers may also pose a risk to classified computer systems. Havoc could be wreaked by erasing hard drives, tape or other forms of backup, physically destroying equipment, or introducing malicious code. HRP designation of systems administrator positions means that these personnel are evaluated to detect changes in attitude and motivation or identify other reliability concerns.

Including systems administrators in the HRP would expose them to the same continuous observation process experienced by other workers who have access to classified information. Including systems administrator positions in the HRP would also fulfill a recommendation in the Mies ${ }^{1}$ review of NNSA security to "Require screening of all sensitive e-network administrators as part of the Human Reliability Program.”

\section{Intelligence/counterintelligence}

Individuals involved in intelligence or counterintelligence operations ask the question, "Who is watching the watchers?" Intelligence and counterintelligence personnel have access to highly

${ }^{1}$ Mies, Richard W. et al. NNSA Security: An Independent Review, Sage Systems Technologies, LLC, and LMI, April 2005. 
sensitive information. Additionally, these personnel may be in direct contact with those involved in espionage activities. The vulnerability of intelligence officers revolves around both the quality of their work and their access to sensitive information and materials that may be valuable to other nations and groups.

Like the other proposed additions to the positions designated in the HRP, employees in intelligence/counterintelligence positions need to be reliable. Their jobs require concentration and attention to detail, which can be negatively influenced by a variety of mental and physical conditions and other circumstances.

As with any worker, changes in health status of the individual or loved ones, family or other domestic problems, even changes in the job can produce stress. The ability of the individual to manage that stress and perform job tasks in a safe and secure manner may be challenged. That same stress can lead to alcohol or drug abuse as a form of self medication.

Because the principal purpose of the intelligence/counterintelligence program should be a comprehensive and proactive approach to protecting DOE materials and information, it is imperative that intelligence and counterintelligence personnel be included in the HRP. A regular and systematic assessment can ensure that the workers are focused on their tasks while in compliance with rules and procedures.

\section{Biological and chemical program personnel}

Several DOE facilities have robust programs to detect chemical and biological agents that could be released into the environment. The personnel who work with these detection systems and have access to chemical and biological agents are currently not covered by a DOE program but rather are covered by facility-administered programs closely patterned after the HRP. ${ }^{*}$ These programs incorporate almost all facets of the DOE HRP but have no headquarters policy oversight or support. Personnel included in these programs also have no avenue for appealing certification or revocation decisions. These characteristics necessarily limit the effectiveness of the local programs and may pose a liability risk, both to individual facilities and the DOE.

\footnotetext{
* An example is the Lawrence Livermore National Laboratory Select Agent Human Reliability Program implemented in March 2005.
} 
One critical difference between the local programs and the HRP is that local certification must be approved by another government agency initially and at five-year intervals thereafter, whereas the HRP has an annual DOE certification requirement. It is therefore proposed that these local programs be eliminated and personnel in positions currently covered under them be assimilated into the HRP population. This would eliminate processing these individuals in separate but very similar programs, ensure headquarters oversight, and provide participants with the review and appeal rights afforded HRP-certified individuals.

Protecting the nation from chemical and biological threats should include ensuring the reliability of those who design and deploy the detection systems or have access to the biological/chemical agents. These individuals represent safety and security concerns that can be considered as significant as those posed by HRP-certified personnel who design, protect, and transport weapons systems. It is therefore proposed that individuals who have knowledge of or access to chemical and biological agents or detection systems be HRP certified.

\section{Review of current positions}

A review should be conducted of positions currently requiring HRP certification and some current HRP positions excluded from the program. The most obvious are the Security Police Officer (SPO) positions, which are covered by 10 CFR Part 1046, Physical Protection of Security Interests. This regulation thoroughly delineates physical, mental, and training requirements for SPOs and has more stringent suitability requirements than the HRP. The one exception to this is the access authorization requirement. Currently all HRP candidates and HRP-certified individuals must have a DOE “Q” access authorization. Under 10 CFR \$1046.14, some SPOs are not required to maintain a “Q” level access authorization:

Security police officer personnel who have access to Category I or II quantities of special nuclear material (SNM) will be "Q" cleared. The specific level of access authorization for each duty assignment shall be designated by the site security organization and approved by the Head of the Field Element. Security police officers shall possess a minimum of an "L" or DOE Secret access authorization. Security police officers possessing less than "Q" access authorization shall not be assigned to offensive positions or duties where fully automatic firearms are required.

All SPOs having access to Category I or II SNM still require a "Q" access authorization, which is one of the HRP requirements and supports the security interests of DOE. 


\section{Conclusion}

All positions at DOE facilities should be carefully reviewed and evaluated to assess the relationship to not only the insider risk, but also the risk to the overall national security. It is highly recommended that, in addition to those positions listed in 10 CFR $\$ 712.10$, the rule should be modified to include individuals assigned to, or applying for the following positions:

- Those affording access to information contained in weapons data categories Sigma 14 and 15; Sigmas 1-13 should also be evaluated for inclusion.

- Executive protection services agents.

- Designated systems administrators.

- Intelligence/counterintelligence personnel.

- Those with access to, (1) chemical and biological release detection systems, and/or (2) chemical and biological agents.

It would appear that the reliability of SPOs is adequately addressed in another regulation, $10 \mathrm{CFR}$ Part 1046, and therefore consideration should be given to removing this group from the HRP. Although diverse, individuals in the proposed HRP positions may pose a unique insider risk as well as a risk to the safety and security of other personnel, DOE facilities, and the national security. Therefore, DOE should evaluate these positions for inclusion in the DOE HRP (10 CFR \$712.10). 